\title{
Analytical investigation of the atmospheric radiation limits in semigray atmospheres in radiative equilibrium
}

\author{
By TONI PUJOL* and GERALD R. NORTH, Department of Atmospheric Sciences, Texas A\&M University, \\ College Station, Texas 77843-3150, USA
}

(Manuscript received 8 April 2002; in final form 25 February 2003)

\begin{abstract}
We model the wavelength-dependent absorption of atmospheric gases by assuming constant mass absorption coefficients in finite-width spectral bands. Such a semigray atmosphere is analytically solved by a discrete ordinate method. The general solution is analyzed for a water vapor saturated atmosphere that also contains a carbon dioxide-like absorbing gas in the infrared. A multiple stable equilibrium with a relative upper limit in the outgoing long-wave radiation is found. Differing from previous radiative-convective models, we find that the amount of carbon dioxide strongly modifies the value of this relative upper limit. This result is also obtained in a gray (i.e., equal absorption of radiation at all infrared wavelengths) water vapor saturated atmosphere. The destabilizing effect of carbon dioxide implies that massive carbon dioxide atmospheres are more likely to reach a runaway greenhouse state than thin carbon dioxide ones.
\end{abstract}

\section{Introduction}

The purpose of simple physically based models is to provide good analogs of the basic processes that govern the climate. This thermodynamic viewpoint differs conceptually from the atomistic approach used in more complex models, such as general circulation ones, which are focused on simulating the system by describing the maximum possible number of mechanisms that occur in the real climate. Among simple models, those of radiative equilibrium have been widely used in order to examine the general principles governing a planetary atmosphere. As pointed out by Goody and Yung (1989), these types of models represent the first step in the study of atmospheres of other planets (Gierasch and Goody, 1970), stellar atmospheres (Chandrasekhar, 1960) and sensitivity of the Earth's atmosphere (Ide et al., 2001). The main

\footnotetext{
${ }^{*}$ Correspoding author. Current address: Department of Physics, Universitat de Girona, Campus Montilivi, 17071 Girona, Catalonia, Spain.

e-mail: toni.pujol@udg.es
}

feature of such models is the exclusion of vertical nonradiative energy fluxes. Therefore, the assumption of radiative equilibrium holds at every level of the atmosphere, from which simple solutions can be extracted. Thus, sensitivity analyses regarding the different parameters used in the model can be easily performed, with the aim of shedding light on the planetary climatic behavior.

Nevertheless, reasonable doubts about the relevance of radiative equilibrium solutions arise when noting the disagreement of such model solutions with the observed Earth's atmospheric structure (e.g., Goody and Yung, 1989). Although the inclusion of a convective lower layer leads to more realistic results (see Ramanathan and Coakley, 1978), the heuristic way of introducing such an adjustment does not dispel the concerns of neglecting dynamical effects (Goody and Yung, 1989). Currently, elaborate radiativeconvective models with physically based parameterizations of convective effects are of great interest for analyzing the climate system (see Liou, 1992), whereas simpler versions, such as the gray approach (i.e., absorption of radiation independent of wavelength), 
are mainly devoted to academic purposes (see, e.g., Houghton, 1986).

However, Weaver and Ramanathan (1995) showed that the addition of a transparent spectral window in the absorption spectrum could resolve some obvious deficiencies of gray radiative equilibrium models (namely, non-zero discontinuity of temperatures at the surface and ground temperature increasing without bound as the amount of the absorbing gas is allowed to increase). The present paper aims to extend the initial work carried out by Weaver and Ramanathan (1995) by deducing a general analytical expression for the temperature profile in a model atmosphere with multiple gases and constant mass absorption coefficients in finite-width spectral bands. Note that our purpose is not to reproduce quantitatively the absorption of infrared radiation, since detailed non-gray models that require numerical solutions have been widely used to analyze the behavior of planetary atmospheres (e.g., Pollack, 1971; Abe and Matsui, 1988). Instead, our aim is to develop an analytical model with a reasonable (although not extremely accurate) treatment of infrared absorption that may be used for providing qualitative answers of those problems difficult to analyze with more elaborate models.

Sagan (1972), Henderson-Sellers and Meadows (1976) and others did apply very simple non-gray models in order to gain an insight into the long-term evolution of planetary atmospheres. The model shown in section 2 substantially improves the one applied in these pioneering studies, since it is deduced from a discrete ordinate method that avoids some inconsistencies found in these former studies where the outgoing long-wave radiation flux in the opaque regions was expressed in terms of the blackbody radiation emitted at the skin temperature (or, equivalently, at the effective temperature divided by $2^{1 / 4}$ ). This procedure approximately accounted for half of the outgoing long-wave radiation only (the contribution due to the emission at top of the atmosphere), since it ignored the contribution associated to the transmission of the upward radiation flux that comes for lower layers (see, e.g., Goody and Yung, 1989; Weaver and Ramanathan, 1995; Visconti, 2001).

As a particular application of the general expression obtained in section 2, in section 3 we analyze the effects of carbon dioxide on the atmospheric radiation limits that arise in a water vapor saturated atmosphere. Here we find a non-linear effect of the amount of carbon dioxide on the atmospheric radiation limit that may be of importance for the long-term evolution of planetary atmospheres. In addition, the important stabilizing effect of the atmospheric window is also shown. Finally, the main conclusions of the present paper are shown in section 4 , where we also discuss the severe limitations of our model atmosphere.

\section{Semigray atmosphere in radiative equilibrium}

In planetary atmospheres, radiation fluxes can be split into two terms, short-wave incoming solar radiation and long-wave planetary emission, which (in most cases) can be separately analyzed. The radiative equilibrium assumption applied to the model atmosphere ignores convective fluxes, which implies a balance between short- and long-wave radiative fluxes at any vertical level, including the surface. For the sake of simplicity, we also assume a cloudless plane-parallel atmosphere completely transparent to short-wave radiation, which means that the ground is the only level that absorbs solar radiation. Although this is not the case for the current Earth's atmosphere (e.g., there is a strong short-wave absorption by ozone in the stratosphere), the transparent approach to sunlight has been usually applied in order to solve simple radiative equilibrium models (see, e.g., Liou, 1992).

We solve the transfer of long-wave radiation throughout the model atmosphere by using the following two boundary conditions. At the surface, the upward long-wave flux follows the Planck function at the ground temperature. At the top of the atmosphere (TOA), the downward long-wave flux is zero. Note that both boundary conditions apply monochromatically. In addition, we assume a source function for the infrared atmospheric emission equal to the Planck function, which implies local thermodynamic equilibrium condition.

From the previous physical assumptions, the solution for the vertical temperature profile by using constant mass absorption coefficients in each one of the $n$ finite-width spectral bands in which we have divided the infrared spectrum is as follows (see Appendix):

$$
\sigma T^{4}=\frac{Q a}{2} \frac{\sum_{i=1}^{n}\left[\kappa_{i} \beta_{g i} \frac{\left(1+D \tau_{i}\right)}{\left(2+D \tau_{i}^{*}\right)}\right]}{\left\{\sum_{i=1}^{n} \kappa_{i} \beta_{i}\right\}\left\{\sum_{i=1}^{n}\left[\frac{\beta_{g i}}{\left(2+D \tau_{i}^{*}\right)}\right]\right\}},
$$


where $\sigma$ is the Stefan-Boltzmann constant, $T$ is the atmospheric temperature, $Q a$ is the absorbed short-wave radiation at the surface ( $Q$ is the solar constant divided by four and $a$ is the co-albedo), $D=3 / 2, \kappa_{i}$ is the volume absorption coefficient of the $i$-th spectral band, and $\beta_{i}$ is the effective width of the $i$-th spectral band, defined as

$\beta_{i}=\frac{\int_{v_{l i}}^{v_{u i}} B_{v} \mathrm{~d} v}{\int_{0}^{\infty} B_{v} \mathrm{~d} v}$,

where $v_{l i}$ and $v_{u i}$ are the lower and upper frequency limits of the $i$-th spectral band, and $B_{v}$ is the Planck function. Note that the summation over all $\beta_{i}$ is equal to 1 . In eq. (1), $\beta_{g i}$ refers to the effective width of the $i$-th spectral band at the ground, whereas the optical depth at height $z$ (assuming TOA at $z=\infty$ ) of the $i$-th spectral band $\tau_{i}$ follows

$\tau_{i}=\int_{z}^{\infty} \kappa_{i} \mathrm{~d} z^{\prime}=\int_{z}^{\infty} k_{i} \rho_{a i} \mathrm{~d} z^{\prime}$,

where $k_{i}$ and $\rho_{a i}$ are the constant mass absorption coefficient and the absorbing gas density of the $i$-th spectral band, respectively. The optical thickness of the $i$-th spectral band $\tau_{i}^{*}$ follows from eq. (3) at $z=0$. Note that we allow for the absorption of multiple gases in the same spectral band.

Finally, the ground temperature $T_{g}$ reads (see Appendix)

$\sigma T_{g}^{4}=\frac{Q a}{2} \frac{1}{\sum_{i=1}^{n}\left[\beta_{g i}\left(2+D \tau_{i}^{*}\right)^{-1}\right]}$.

Note that the air temperature at the surface obtained from eq. (1) is lower than the ground temperature, eq. (4), by a finite amount. Such a discontinuity at the boundaries is a common feature in pure radiative equilibrium atmospheres (Goody and Yung, 1989).

\section{Atmosphere with water vapor and carbon dioxide absorbing gases}

\subsection{Analytical solution}

Here we analyze a water vapor saturated atmosphere with a carbon dioxide-like absorbing gas in the infrared. For water vapor, we assume a constant mass absorption coefficient at infrared wavelengths outside the atmospheric window. This window ranges from
8 to $12 \mu \mathrm{m}$ (Kondratyev, 1969). For carbon dioxide, we only take the strong band centered at $15 \mu \mathrm{m}$ into account (from 13 to $17.6 \mu \mathrm{m}$ in wavelength; Lindzen et al., 1982). Thus, the infrared spectrum is divided into five bands ( $n=5$; see Fig. 1). Band 1 corresponds to $\lambda>8 \mu \mathrm{m}$. Band 2 ranges from $8 \leq \lambda \leq 12 \mu \mathrm{m}$ (water vapor atmospheric window). Band 3 ranges from $12<\lambda<13 \mu \mathrm{m}$. Band 4 ranges from $13 \leq \lambda \leq$ $17.6 \mu \mathrm{m}$ (carbon dioxide band). Band 5 corresponds to $\lambda>17.6 \mu \mathrm{m}$. Band 2 is totally transparent. Bands 1 , 3 and 5 assume absorption by water vapor only. Band 4 assumes absorption by carbon dioxide and water vapor. Then, eq. (4) reads

$$
\begin{aligned}
& \sigma T_{g}^{4}=Q a \\
& \quad \times \frac{\left(2+D \tau_{w}^{*}\right)\left[2+D\left(\tau_{w}^{*}+\tau_{c}^{*}\right)\right]}{\left(2+\beta_{g w} D \tau_{w}^{*}\right)\left[2+D\left(\tau_{w}^{*}+\tau_{c}^{*}\right)\right]-2 \beta_{g c} D \tau_{c}^{*}},
\end{aligned}
$$

where $\tau_{w}^{*}$ is the optical thickness due to the absorption by water vapor in bands 1 and $3-5$, and $\tau_{c}^{*}$ is the optical thickness due to the absorption by carbon dioxide in band 4 . Since we assume a purely additive overlapping for both water vapor and carbon dioxide, the net optical thickness in band 4 is $\tau_{w}^{*}+\tau_{c}^{*}$. Although the model here developed may use different values of the mass absorption coefficient $k_{w}$ in different spectral

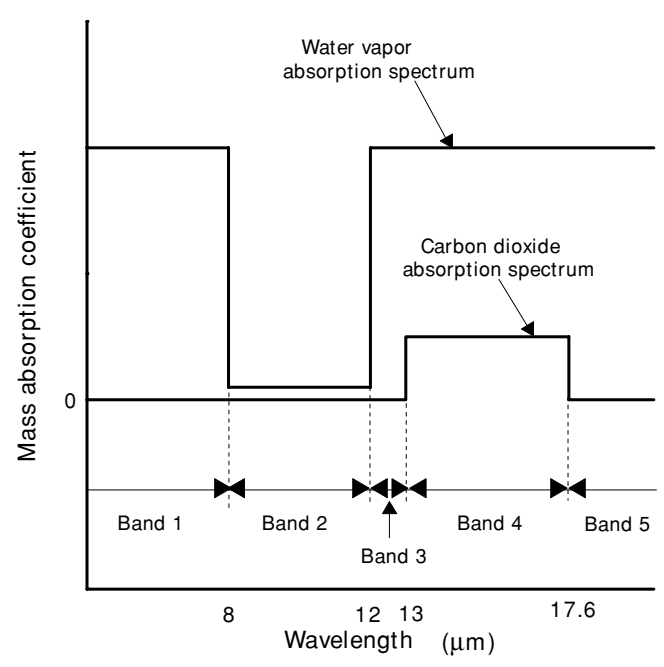

Fig. 1. The semigray model uses five infrared bands with different but constant mass absorption coefficients. The limits of the carbon dioxide band follow from Lindzen et al. (1982). Gray atmospheres in the infrared spectrum use mass absorption coefficients independent of wavelength. 
bands, eq. (5) assumes the same value of $k_{w}$ for bands 1 and 3-5 (i.e., those bands outside the atmospheric window). This is a very crude approximation to the infrared spectrum of water vapor absorption, since it greatly varies in wavelength (see, e.g., Kasting et al., 1984). However, variations of the absorption between bands outside the atmospheric window are substantially smaller than those between the same bands and the absorption within the atmospheric window. Therefore, the approximation employed here represents a first approximation to the actual non-gray absorption of water vapor, being reasonable for qualitative analyses. In eq. (5), $\beta_{g w}$ and $\beta_{g c}$ are the effective widths of the water vapor atmospheric window and carbon dioxide spectral band at the ground temperature, respectively.

The spectral-mean optical thickness is defined as the Planck mean (see Goody and Yung, 1989):

$$
\tau^{*}=\frac{\int_{0}^{\infty} \tau_{\nu}^{*} B_{v} \mathrm{~d} v}{\int_{0}^{\infty} B_{v} \mathrm{~d} v}=\sum_{i=1}^{n} \tau_{i}^{*} \beta_{i},
$$

where $\tau_{v}^{*}$ is the optical thickness at frequency $v$ and $i$ refers to the $i$-th spectral band. Note that the last equality in eq. (6) follows from eq. (2) and the assumption of uniform values of $\tau_{v}^{*}$ at each one of the $n$ spectral bands in which we have divided the infrared spectrum. From eq. (6), the optical thickness of the case here analyzed is

$\tau^{*}=\tau_{w}^{*}\left(1-\beta_{g w}-\beta_{g c}\right)+\left(\tau_{w}^{*}+\tau_{c}^{*}\right) \beta_{g c}$.

The optical thickness for the Earth's current state is a function of the surface temperature since water vapor coexists with its liquid phase at the surface. The equilibrium condition between both vapor and liquid phases restricts the amount of water vapor in the atmosphere and, hence, limits the possible values of optical thickness. This effect has been extensively analyzed in gray and non-gray models (see, e.g., Komabayasi, 1967; Ingersoll, 1969; Kasting, 1988; Nakajima et al., 1992; Pierrehumbert, 1995). For an atmosphere with constant mass fraction of the absorbing gas (as expected in current Earth's stratosphere), we find (see Ingersoll, 1969)

$\tau_{w}^{*}=\frac{k_{w} e_{w s} m_{w}}{g \bar{m}}$,

$\tau_{c}^{*}=\frac{k_{c} p_{c} m_{c}}{g \bar{m}}$,

where $k_{w}\left(=0.01 \mathrm{~m}^{2} \mathrm{~kg}^{-1}\right)$; Ingersoll, 1969; Nakajima et al., 1992) and $k_{c}\left(=0.05 \mathrm{~m}^{2} \mathrm{~kg}^{-1}\right)$ are the constant mass absorption coefficients for water vapor outside the atmospheric window and carbon dioxide in its single absorbing band, respectively. Note that the mass absorption coefficient $k$ is an intrinsic radiative property of the absorber and it can be a function of temperature and pressure (effects here ignored). The $k_{c}$ value has been chosen to produce the expected climate sensitivity for Earth's current conditions (ground temperature variation on the order of $2 \mathrm{~K}$ for doubling the present atmospheric amount of carbon dioxide). In eq. (7a) $e_{w s}$ is the partial pressure of water vapor at the surface. In consistency with the vapor-liquid equilibrium condition, we assume complete saturation at the surface. The saturation vapor pressure $e_{w s}$ is a function of the air temperature at the surface $T_{a s}$ and it may be obtained from (see Bohren and Albrecht, 1998)

$$
\begin{aligned}
e_{w s}\left(T_{a s}\right)= & 611 \exp \left[6808\left(273^{-1}-T_{a s}^{-1}\right)\right. \\
& \left.-5.09 \ln \left(T_{a s} / 273\right)\right]
\end{aligned}
$$

In comparison with the steam tables provided by Irvine and Liley (1984), eq. (8) differs less than a $6.3 \%$ for temperatures lower than $450 \mathrm{~K}$, which is reasonable enough for our qualitative analyses.

In eq. (7b) we assume that the partial pressure of carbon dioxide $p_{c}$ is independent of the surface temperature. Earth's values suggest $p_{c} \approx 54 \mathrm{~Pa}$ (amount of carbon dioxide $\approx 353$ ppmv; see Hartmann, 1994). In eqs. (7a) and (7b), $g$ is the acceleration due to gravity $\left(9.8 \mathrm{~m} \mathrm{~s}^{-2}\right)$, and $m_{w}, m_{c}$ and $\bar{m}$ are the molecular weights of water vapor $\left(18 \times 10^{-3} \mathrm{~kg} \mathrm{~mol}^{-1}\right)$, carbon dioxide $\left(44 \times 10^{-3} \mathrm{~kg} \mathrm{~mol}^{-1}\right)$ and mean atmosphere. The value for $\bar{m}$ is obtained from the partial pressures and molecular weights of water vapor, carbon dioxide and dry air without carbon dioxide.

From the above, the atmospheric temperature obtained from eq. (1) reads

$\sigma T^{4}=Q a \frac{\kappa_{w}\left(1-\beta_{g w}-\beta_{g c}\right)\left(1+D \tau_{w}\right)\left[2+D\left(\tau_{w}^{*}+\tau_{c}^{*}\right)\right]+\left(\kappa_{w}+\kappa_{c}\right) \beta_{g c}\left(2+D \tau_{w}^{*}\right)\left[1+D\left(\tau_{w}+\tau_{c}\right)\right]}{\left\{\left(2+\beta_{g w} D \tau_{w}^{*}\right)\left[2+D\left(\tau_{w}^{*}+\tau_{c}^{*}\right)\right]-2 \beta_{g c} D \tau_{c}^{*}\right\}\left[\kappa_{w}\left(1-\beta_{w}\right)+\kappa_{c} \beta_{c}\right]}$ 
where $\kappa_{w}$ and $\kappa_{c}$ are the volume absorption coefficients for water vapor and carbon dioxide. These values are directly related to the mass absorption coefficients $k_{w}$ and $k_{c}$ through the gas densities [see, e.g., eq. (3)]. Here we assume water vapor, carbon dioxide and dry air without carbon dioxide as ideal gases. In eq. (9), $\beta_{w}$ and $\beta_{c}$ are the effective widths of the water vapor atmospheric window (band 2) and carbon dioxide absorbing band (band 4) at temperature $T$. In addition, $\tau_{w}$ and $\tau_{c}$ are the optical depth due to water vapor (in either band 1, 3 or 5) and carbon dioxide (in band 4) absorbing gases, respectively.

Note that eqs. (5) and (9) revert to the semigray solution found by Weaver and Ramanathan (1995) for $\tau_{c}=\tau_{c}^{*}=0=\kappa_{c}$ (i.e., atmosphere without carbon dioxide). In addition, for $\beta_{w}=\beta_{g w}=0=\beta_{c}=\beta_{g c}$, eqs. (5) and (9) revert to the gray solution, as they should.

\subsection{Atmospheric radiation limits}

For simplicity, here we use constant values for $\beta_{w}$ $\left(=\beta_{g w}=0.2488\right)$ and $\beta_{c}\left(=\beta_{g c}=0.1435\right)$, which correspond to the mean values of $\beta_{w}(T)$ and $\beta_{c}(T)$ defined as in eq. (2) with the spectral limits discussed in section 3.1 (Fig. 1), for the 200-600 K temperature range. Then, and for a fixed value of $Q a$, eq. (9) at the surface is a function of $T_{a s}$ only. The solution is obtained by applying Brent's method for finding the root of a function (see Press et al., 1994). The value of the surface temperature $T_{a s}$ is then used in eq. (5) to obtain the ground temperature $T_{g}$.

Figure 2 shows the absorbed short-wave radiation $Q a$ as a function of the ground temperature $T_{g}$ for atmospheres with different carbon dioxide amounts. Note that case A ignores the carbon dioxide. In this case, we find three states that satisfy the vapor-liquid equilibrium condition with $Q a=360.7 \mathrm{~W} \mathrm{~m}^{-2}$ (a value that refers to tropical regions for Earth's current conditions). The spectral-mean optical thickness $\tau^{*}$ for these three states is $0.017\left(T_{g} \approx 283.3 \mathrm{~K}\right), 0.78\left(T_{g} \approx\right.$ $312 \mathrm{~K})$ and $148\left(T_{g} \approx 398 \mathrm{~K}\right)$.

Following the classical criterion applied in simple climate models (see, e.g., North et al., 1981), branches with positive slopes (i.e., $\partial Q a / \partial T_{g}>0$ ) in Fig. 2 refer to stable climates. In contrast, climate states found on branches with negative slopes (i.e., $\partial Q a / \partial T_{g}<0$ ) are unstable (i.e., physically unrealistic). The relative radiation limit found in Fig. 2 arises due to the conflict between the amount of absorbing gas required by the vapor-liquid equilibrium condition and that required by the radiative equilibrium one (see, e.g., Nakajima et al., 1992). The stable branch obtained at high values of ground temperature arises due to the existence of the transparent window in the absorption spectrum of water vapor, which leads to a finite value for $T_{g}$ in the optically thick limit $[\approx 400 \mathrm{~K}$ for $Q a=360.7 \mathrm{~W}$ $\mathrm{m}^{-2}$ from eq. (5) and $\tau_{w}^{*} \rightarrow \infty$ ]. On such a branch, most of the outgoing long-wave flux escapes to space through the atmospheric window since other spectral regions have become completely opaque. Note also that the two stable branches obtained in Fig. 2 lead to an hysteresis-like cycle on the ground temperature to changes in the absorbed solar radiation $Q a$ (not found in gray atmospheres).

Case B in Fig. 2 uses a surface partial pressure for carbon dioxide similar to Earth's current value $\left(p_{c} \approx\right.$ $54 \mathrm{~Pa}$ ). However, the ground temperature for the stable state with the observed mean value of the absorbed solar radiation in a clear-sky atmosphere $(\approx 260 \mathrm{~W}$ $\mathrm{m}^{-2}$; Stephens and Greenwald, 1991) is lower than that expected. A higher value would be obtained by lessening the saturation condition at the surface and using a fixed value of relative humidity $(<100 \%)$. Of course, far more important are the effects of convective fluxes, here completely ignored.

In Fig. 2 we do not include results for massive carbon dioxide atmospheres $\left(p_{c} \gg 10^{4} \mathrm{~Pa}\right)$ since our simple model would surely lead to wrong solutions, since Rayleigh scattering (here ignored) is of particular importance in thick carbon dioxide atmospheres (Kasting, 1988). In addition, the pressure-broadening effects would be considerable (i.e., we should impose a variation of the width of the atmospheric bands defined in Fig. 1 in terms of pressure). Finally, condensation of carbon dioxide may occur at such high pressures, and here we ignore this process. These effects have been taken into account by several authors in the study of the particular conditions of early Earth and Venus (e.g., Kasting and Ackerman, 1986; Abe and Matsui, 1988; Kasting, 1991). Note that our purpose here is to help understand the effect of carbon dioxide on the atmospheric radiation limits by means of an analytically solvable model.

We also point out that pressure-broadening effects might be important for the absorption lines of water vapor at high temperatures. In this case, the amount of water vapor in the atmosphere is so high that the atmospheric pressure substantially increases. This effect would reduce the width of the atmospheric window, implying a tendency of the semigray atmosphere to become gray. However, planetary atmospheres with 


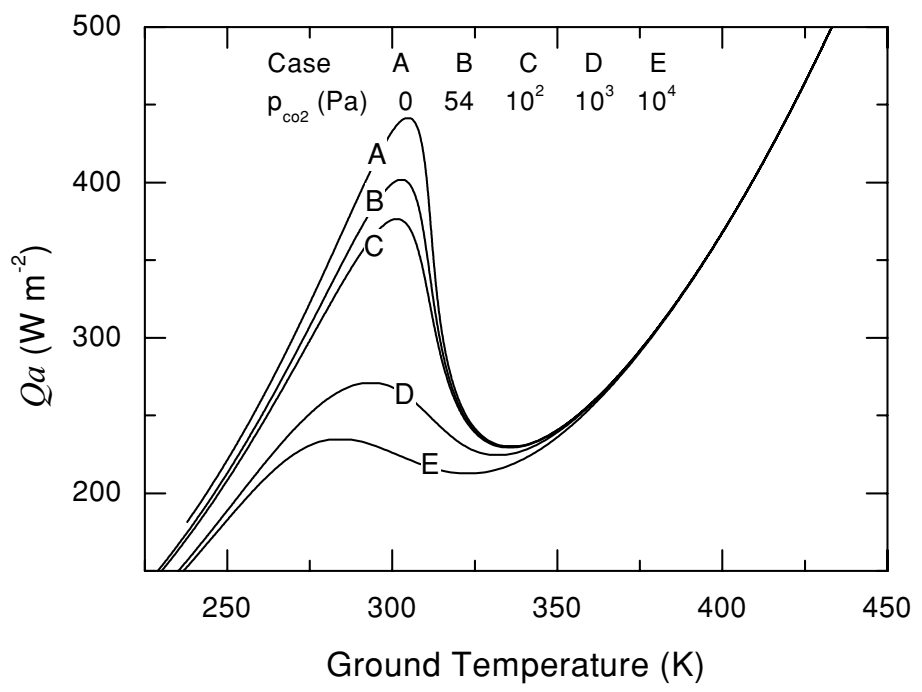

Fig. 2. Absorbed solar radiation $Q a$ as a function of the ground temperature $T_{g}$ for a water vapor saturated atmosphere with different amounts of carbon dioxide (measured in carbon dioxide surface partial pressure). The water vapor-like absorbing gas contains a single totally transparent atmospheric window ranging from 8 to $12 \mu \mathrm{m}$ in wavelength. The carbon dioxide band ranges from 13 to $17.6 \mu \mathrm{m}$ in wavelength (see Fig. 1).

absorbing gases with large atmospheric windows may be better represented by the semigray case for a large range of surface temperatures, as discussed in section 4.

From Fig. 2 we find that carbon dioxide strongly modifies the relative atmospheric radiation limit obtained from both vapor-liquid and radiative equilibrium conditions. This result is not found in radiativeconvective models (see Kasting, 1988), most likely because the radiation limit obtained here is an upper bound for the radiation limits that arise when convective processes are included (see the discussion in Nakajima et al., 1992).

The effect of carbon dioxide on the relative runaway greenhouse point is highly non-linear. The relative radiation limit substantially decreases from $p_{c}=10^{2}$ to $10^{3} \mathrm{~Pa}$ (compare cases $\mathrm{C}$ and $\mathrm{D}$ in Fig. 2). In contrast, the variation of the relative radiation limit from $p_{c}=$ $10^{3}$ to $10^{4} \mathrm{~Pa}$ is not so big (see cases D and E in Fig. 2). This type of behavior has been already been found for the solar constant required to maintain a given value of surface temperature in a radiative-convective model (see Kasting, 1988).

Figure 3 shows the ground temperature as a function of the partial pressure of carbon dioxide for the maximum values of absorbed solar radiation (i.e., $a=$ 1) for early Earth ( $240 \mathrm{~W} \mathrm{~m}^{-2}$ ), current Earth (340 W $\mathrm{m}^{-2}$ ), and early Venus (458 $\mathrm{W} \mathrm{m}^{-2}$ ) (Abe and Matsui, $1988)$. Figure 3 suggests a very warm solution in early Venus $\left(p_{c} \gg 10^{4} \mathrm{~Pa}\right)$ mainly due to the effect of carbon dioxide on the lessening of the value of the relative radiation limit. As stated above, at very high values of surface temperature, the assumption of a fully transparent window would break down since the absorption of water dimes within the atmospheric window would be important. Then, the solution would tend to the gray atmosphere that does not show the second stable branch at high values of surface temperature shown in Fig. 2, so the temperature would run away. For early Earth conditions, Fig. 3 also suggests a very warm atmosphere for a massive amount of carbon dioxide. However, with a small albedo (i.e., $Q a<240 \mathrm{~W} \mathrm{~m}^{-2}$ ), even atmospheres with high amounts of carbon dioxide would attain a moderate temperature (see Fig. 2). We point out that the results obtained for an acceleration of gravity as that expected for Venus $\left(8.9 \mathrm{~m} \mathrm{~s}^{-2}\right)$ do not modify the previous discussion.

For completeness, we show in Fig. 4 the results for a model atmosphere with gray absorption for water vapor and semigray for carbon dioxide. As in Figs. 2 and 3, we assume that water vapor is saturated at the surface. In comparison with Fig. 2, the second stable branch at high values of surface temperature does not appear in Fig. 4, as discussed above. Thus, $T_{g}$ is 


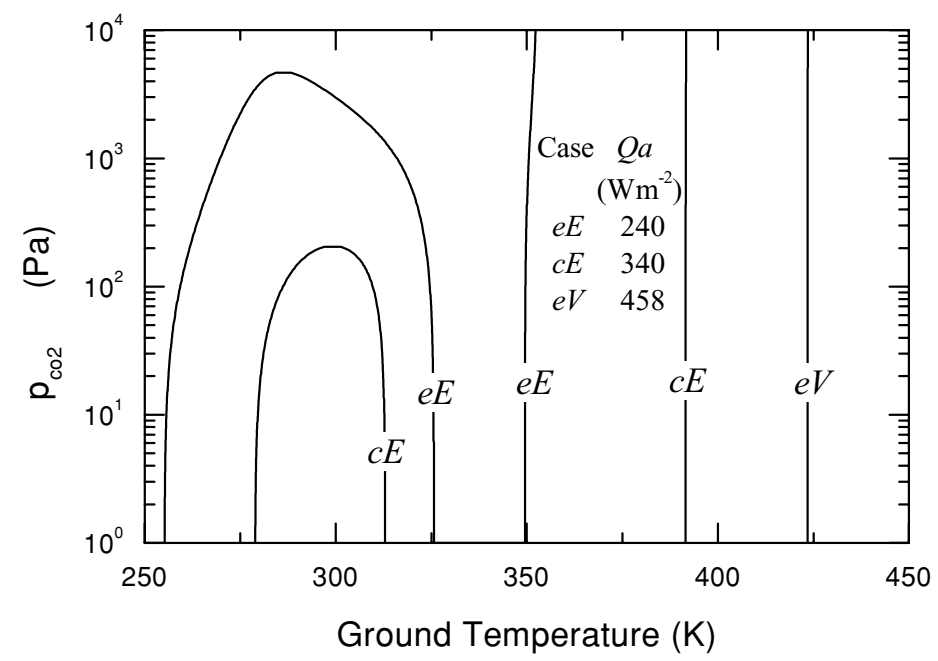

Fig. 3. Ground temperature $T_{g}$ vs. partial pressure of carbon dioxide for a water vapor saturated atmosphere with constant values of absorbed solar radiation. The three cases corresponds to the maximum mean solar absorption $(a=1)$ for early Earth $e E\left(240 \mathrm{~W} \mathrm{~m}^{-2}\right)$, current Earth $c E\left(340 \mathrm{~W} \mathrm{~m}^{-2}\right)$, and early Venus $e V\left(458 \mathrm{~W} \mathrm{~m}^{-2}\right)$. The water vapor-like absorbing gas contains a single totally transparent atmospheric window ranging from 8 to $12 \mu \mathrm{m}$ in wavelength. The carbon dioxide band ranges from 13 to $17.6 \mu \mathrm{m}$ in wavelength.

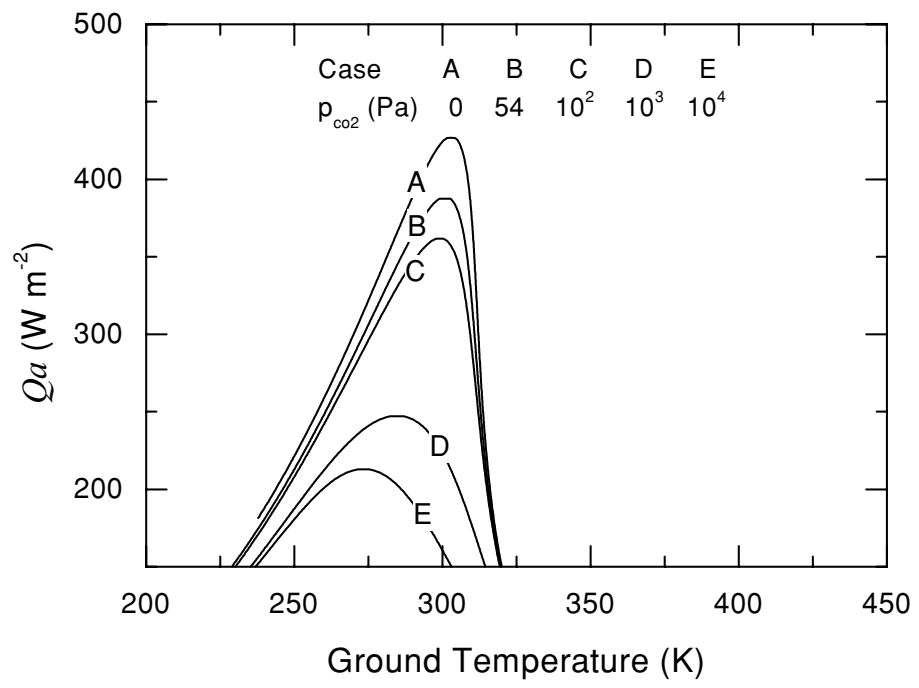

Fig. 4. As in Fig. 2 except for a gray absorption of water vapor. The carbon dioxide band ranges from 13 to $17.6 \mu \mathrm{m}$ in wavelength.

unbound as the optical thickness increases. In this case, there is an absolute radiation limit of value $Q a_{r g}$. For values of the absorbed solar radiation $Q a$ higher than $Q a_{r g}$ the vapor-liquid equilibrium condition cannot hold and the liquid phase evaporates entirely (see, e.g., Komabayasi, 1967; Ingersoll, 1969). Therefore, $Q a_{r g}$ is known as the runaway greenhouse point. Note that this is not the case in a semigray atmosphere with a transparent window, since the new warm stable branch is unbounded (i.e., the vapor-liquid equilibrium still holds in the second stable branch at high values of surface temperature shown in Fig. 2). This shows the 
importance of a transparent atmospheric window on generating a second stable branch.

\section{Conclusions}

We have analytically solved the vertical temperature profile for a cloudless, transparent to sunlight, radiative equilibrium atmosphere with constant mass absorption coefficients in finite-width spectral bands. The solution for a water vapor-like absorbing atmosphere assumes a single transparent window in the infrared, and reverts to the expressions obtained by Weaver and Ramanathan (1995) from a broadband analysis.

We have applied the general expression of our model atmosphere to the particular case of a water vapor saturated atmosphere with a carbon dioxide-like absorbing gas. Our model atmosphere, however, contains very restrictive assumptions that limit its applicability. Besides neglecting convective processes, atmospheric absorption of sunlight and cloud formation (which are serious limitations of the present model), we also ignore possible phase changes in carbon dioxide. Although the condensation of carbon dioxide at the surface does not occur for the values found in Figs. 2-4, the possible formation of carbon dioxide clouds is neglected.

The consistency between the vapor-liquid equilibrium condition for water and the radiative equilibrium condition leads to a relative maximum in the absorbed solar radiation as a function of ground temperature. The effect of the transparent window in the absorption spectrum of water vapor produces a new stable solution at very high values of ground temperature. Nevertheless, water vapor actually absorbs within the atmospheric window. This continuum absorption is expected to increase with surface temperature (see, e.g., Kasting et al., 1984), which implies that the absorption spectrum of water vapor tends to the gray case at high values of ground temperature. Thus, the new stable branch may not be found in a real water-vapor atmosphere. This possibility, however, does not weaken the usefulness of the semigray approach, since in some planetary atmospheres with large atmospheric windows it may help to understand the qualitative behavior of the system. For example, the multiple equilibrium associated to the hysteresis-like cycle found in Titan's atmosphere with more elaborate models (Lorenz et al., 1999) may be a direct consequence of the non-gray absorption here investigated.

Independently of the existence of this new stable branch, we have found that carbon dioxide strongly modifies the relative radiation limit (see Fig. 3; or the runaway greenhouse point, see Fig. 4). This radiation bound decreases as the amount of carbon dioxide increases in non-massive carbon dioxide atmospheres. Note that the vertical lapse rate in gray and semigray optically thick atmospheres may exceed the adiabatic lapse rate. In this case, a convective layer would develop below the radiative equilibrium one. In general, convective conditions may produce an upper radiation bound smaller than the radiation limit obtained from radiative equilibrium conditions (see, e.g., Nakajima et al., 1992). Nevertheless, the very low values of the radiation limits expected for massive carbon dioxide atmospheres from radiative equilibrium conditions may lie below the convective radiation bound. Then the runaway greenhouse effect would be dominated by the stratospheric (i.e., radiative equilibrium) behavior. This case applied to a massive carbon dioxide planet for early Venus conditions would lead to a greenhouse runaway effect. Note that the destabilizing effect of carbon dioxide here shown differs from that based on the high reflectivity of carbon dioxide clouds and their effect on the tropospheric lapse rate found by Caldeira and Kasting (1992) in a simple energy-balance climate model.

\section{Acknowledgments}

Thoughtful comments of the reviewers are greatly appreciated. T. Pujol gratefully acknowledges the support of a Fulbright-Generalitat de Catalunya postdoctoral grant, as well as the partial support of the Ministerio de Ciencia y Tecnología of the Spanish Government under contract REN2000-1621.

\section{Appendix. Eddington's approximation}

The transfer of the specific intensity of radiation $I_{v}$ (energy per unit time, area, solid angle and frequency v) through the atmosphere consists of thermal (i.e., long-wave) radiation originating at the ground that emits at temperature $T_{g}$ and being transferred through the atmosphere above. With the physical assumptions stated in section 2 , the equation that describes the variation of the specific intensity of radiation through a slab of thickness $\mathrm{d} z, z$ the altitude above the ground, follows (e.g., Goody and Yung, 1989)

$\mu \frac{\mathrm{d} I_{v}}{\mathrm{~d} z}=-\kappa_{v}\left(I_{v}-B_{v}\right)$,

where $\mu$ is the cosine of the zenith angle and $\kappa_{v}$ and $B_{v}$ were defined in section 2 . 
In general, $I_{v}$ is a function of frequency, position and direction. Referred to the angular direction, $I_{v}$ can be expanded in a series of Legendre polynomials depending on $\mu$ (Liou, 1992). Since our interest is focused on averaged angular properties (such as radiation fluxes), we truncate the expansion of Legendre polynomials at first order (a procedure known as Eddington's approach)

$I_{v}(z, \mu)=I_{0 v}(z)+I_{1 v}(z) P_{1}(\mu)$,

$P_{1}$ being the first-order Legendre polynomial.

By substituting (A2) into the radiative transfer equation (A1) and integrating over the solid angle we obtain

$\frac{1}{3} \frac{\mathrm{d} I_{1 v}}{\mathrm{~d} z}=-\kappa_{v}\left(I_{0 v}-B_{v}\right)$.

By substituting eq. (A2) into the radiative transfer equation (A1) multiplied by $\mu$ and integrating over the solid angle we obtain

$\frac{\mathrm{d} I_{0 v}}{\mathrm{~d} z}=-\kappa_{v} I_{1 v}$

Note that all the variables and parameters in eqs. (A3) and (A4) may vary with altitude $z$. For a gray atmosphere, $\kappa_{v}$ is independent of frequency, and eqs. (A3) and (A4) lead to similar expressions but for broadband quantities once integrated over all $v$. The solution with such a condition is well known and has become the first step toward understanding atmospheres in radiative equilibrium (e.g., Goody and Yung, 1989).

By applying the classical relationship between radiation fluxes and specific intensity of radiation (e.g., Goody and Yung, 1989), upward $F_{v}^{+}$(towards TOA) and downward $F_{v}^{-}$radiation fluxes within the Eddington approach (A2) are

$F_{v}^{+}=\pi I_{0 v}+\frac{2 \pi}{3} I_{1 v}$,

$F_{v}^{-}=\pi I_{0 v}-\frac{2 \pi}{3} I_{1 v}$.

The net long-wave radiation flux is $F_{v}=F_{v}{ }^{+}-$ $F_{v}^{-}=4 \pi I_{1 v} / 3$. Under monochromatic radiative equilibrium conditions (i.e., the net long-wave radiation flux $F_{v}$ does not vary with height; see Liou, 1992; Gorshkov and Makarieva, 2002), $I_{1 v}$ is constant (i.e., does not vary with height). Then, the integration of eq. (A4) from TOA (i.e., $z=\infty$ ) to $z$ is
$I_{0 v}(z)=I_{1 v} \int_{z}^{\infty} \mathrm{d} z^{\prime} \kappa_{v}\left(z^{\prime}\right)+I_{0 v}(\infty)$.

The boundary condition of zero downward fluxes $F_{v}^{-}$ at TOA implies [see eq. (A5b)]

$I_{0 v}(\infty)=\frac{2}{3} I_{1 v}$

from which eq. (A6) reads

$I_{0 v}(z)=I_{1 v}\left[\frac{2}{3}+\int_{z}^{\infty} \mathrm{d} z^{\prime} \kappa_{v}\left(z^{\prime}\right)\right]$.

At the surface $(z=0)$, eq. (A8) is

$I_{0 v}(0)=I_{1 v}\left[\frac{2}{3}+\int_{0}^{\infty} \mathrm{d} z^{\prime} \kappa_{v}\left(z^{\prime}\right)\right]$.

The boundary condition at the surface requires that the upward long-wave flux (A5a) must be equal to the blackbody emission at the ground temperature

$\pi B_{g v}=F_{v}^{+}(0)=\pi I_{0 v}(0)+\frac{2 \pi}{3} I_{1 v}$,

where $B_{g v}$ is the Planck function at the ground temperature. Substituting eq. (A9) into eq. (A10) and rearranging terms, we obtain

$F_{v}=\frac{4 \pi}{3} I_{1 v}=\frac{2 \pi B_{g \nu}}{\left[2+D \int_{0}^{\infty} \mathrm{d} z^{\prime} \kappa_{v}\left(z^{\prime}\right)\right]}$,

where $D=3 / 2$.

The integration of eq. (A11) over frequencies assuming the infrared spectrum divided into $n$ discrete bands leads to eq. (4)

$\pi B_{g}=\frac{Q a}{2} \frac{1}{\sum_{i=1}^{n}\left[\beta_{g i}\left(2+D \tau_{i}^{*}\right)^{-1}\right]}$,

where $\beta_{g i}$ and $\tau_{i}^{*}$ were defined in section 2. Note that radiative equilibrium conditions require that $F=Q a$. In eq. (A12), $B_{g}$ is the Planck function at the ground temperature.

Under monochromatic radiative equilibrium conditions [see the text below Eq. (A5b)], eq. (A3) leads to

$\kappa_{v}(z) B_{v}(z)=\kappa_{v}(z) I_{0 v}(z)$

By substituting eq. (A8) into eqs. (A13) and (A11), we obtain 
$\kappa_{v}(z) \pi B_{v}(z)=\kappa_{v}(z) \pi B_{g v} \frac{\left[1+D \int_{z}^{\infty} \mathrm{d} z^{\prime} \kappa_{v}\left(z^{\prime}\right)\right]}{\left[2+D \int_{0}^{\infty} \mathrm{d} z^{\prime} \kappa_{v}\left(z^{\prime}\right)\right]}$

The integration of eq. (A14) over frequencies assuming the infrared spectrum divided into $n$-th discrete bands and using eq. (A12) leads to

$$
\pi B=\frac{Q a}{2} \frac{\sum_{i=1}^{n}\left[\kappa_{i} \beta_{g i} \frac{\left(1+D \tau_{i}\right)}{\left(2+D \tau_{i}^{*}\right)}\right]}{\left\{\sum_{i=1}^{n} \kappa_{i} \beta_{i}\right\}\left\{\sum_{i=1}^{n}\left[\frac{\beta_{g i}}{\left(2+D \tau_{i}^{*}\right)}\right]\right\}},
$$

which is eq. (1).

\section{REFERENCES}

Abe, Y. and Matsui, T. 1988. Evolution of an impactgenerated $\mathrm{H}_{2} \mathrm{O}-\mathrm{CO}_{2}$ atmosphere and formation of a hot proto-ocean on Earth. J. Atmos. Sci. 45, 3081-3101.

Bohren, C. F. and Albrecht, B. A. 1998. Atmospheric thermodynamics. Oxford University Press, New York.

Caldeira, K. and Kasting, J. F. 1992. Susceptibility of the early Earth to irreversible glaciation caused by carbon dioxide clouds. Nature 359, 226-228.

Chandrasekhar, S. 1960. Radiative transfer. Dover, New York.

Gierasch, P. and Goody, R. M. 1970. Models of the Venus clouds. J. Atmos. Sci. 27, 224-245.

Goody, R. M. and Yung, Y. L. 1989. Atmospheric radiation. Theoretical basis. 2nd edn., Oxford University Press, New York.

Gorshkov, V. G. and Makarieva, A. M. 2002. Greenhouse effect dependence on atmospheric concentrations of greenhouse substances and the nature of climate stability on Earth. Atmos. Chem. Phys. Discuss. 2, 289-337.

Hartmann, D. L. 1994. Global physical climatology. Academic Press, New York.

Henderson-Sellers, A. and Meadows A. J. 1976. The evolution of the surface temperature of Mars. Planet. Space Sci. 24, 41-44.

Houghton, J. T. 1986. The physics of atmospheres. 2nd edn., Cambridge University Press, New York.

Ide, K., Le Treut, H., Li, Z.-X. and Ghil, M. 2001. Atmospheric radiative equilibria. Part II: bimodal solutions for atmospheric optical properties. Climate Dynamics 18, 2949.

Ingersoll, A. P. 1969. The runaway greenhouse: A history of water on Venus, J. Atmos. Sci. 26, 1191-1198.

Irvine, T. F. and Liley, P. E. 1984. Steam and gas tables with computer equations. Academic Press, San Diego, California.

Kasting, J. F. 1988. Runaway and moist greenhouse atmospheres and the evolution of Earth and Venus. Icarus 74, 472-494.

Kasting, J. F., Pollack, J. B. and Ackerman, T. P. 1984. Response of Earth's atmosphere to increases in solar flux and implications for loss of water from Venus. Icarus 57, 335355.

Komabayasi, M. 1967. Discrete temperatures of a hypothetical planet with the atmosphere and the hydrosphere of one component-two phase system under constant solar radiation. J. Meteorol. Soc. Jpn. 45, 137-139.

Kondratyev, K. Y. A. 1969. Radiation in the atmosphere. Academic Press, New York.

Lindzen, R. S., Hou, A. Y. and Farrell, B. F. 1982. The role of convective model choice in calculating the climate impact of doubling $\mathrm{CO}_{2}$. J. Atmos. Sci. 39, 1189-1205.

Liou, K. N. 1992. Radiation and cloud processes in the atmosphere. Oxford University Press, New York.

Lorenz, R. D., McKay, C. P. and Lunine, J. I. 1999. Analytic investigation of climate stability on Titan: sensitivity to volatile inventory. Planet. Space Sci. 47, 1503-1515.

Nakajima, S., Hayashi, Y-Y. and Abe, Y. 1992. A study on the "runaway greenhouse effect" with a one-dimensional radiative-convective equilibrium model. J. Atmos. Sci. 49, 2256-2266.

North, G. R., Cahalan, R. F. and Coakley, J. A. Jr. 1981. Energy balance climate models. Rev. Geophys. Space Phys. 19, 91-121.

Pierrehumbert, R. T. 1995. Thermostats, radiator fins, and the local runaway greenhouse. J. Atmos. Sci. 52, 17841806.

Pollack, J. B. 1971. A nongrey calculation of the runaway greenhouse: Implications for Venus' past and present. Icarus 14, 295-306.

Press, W. H., Teukolsky, S. A., Vetterling, W. T. and Flannery, B. P. 1994. Numerical Recipes in Fortran. The art of scientific computing. 2nd edn., Cambridge University Press, New York.

Ramanathan, V. and Coakley, J. A. Jr. 1978. Climate modeling through radiative-convective models, Rev. Geophys. Space Phys. 16, 465-489.

Sagan, C. 1972. Earth and Mars: Evolution of atmospheres and surface temperatures. Science 177, 52-56.

Stephens, G. L. and Greenwald, T. 1991. The Earth's radiation budget and its relation to atmospheric hydrology. Part 1: Observations of the clear sky greenhouse effect. J. Geophys. Res. 96, 15 311-15324.

Visconti, G. 2001. Fundamentals of physics and chemistry of the atmosphere. Springer-Verlag, Berlin.

Weaver, C. P. and Ramanathan, V. 1995. Deductions from a simple climate model: Factors governing surface temperature and atmospheric thermal structure. J. Geophys. Res. 100, $11585-11591$.

Tellus 55A (2003), 4 